\title{
IS I I I I insertion sequences of Coxiella burnetii: characterization and use for repetitive element PCR-based differentiation of Coxiella burnetii isolates
}

\author{
Amy M Denison, Herbert A Thompson and Robert F Massung*
}

Address: Coordinating Center for Infectious Diseases, Division of Viral and Rickettsial Diseases, Rickettsial Zoonoses Branch, Centers for Disease Control and Prevention, Atlanta, GA, USA

Email: Amy M Denison - crk6@cdc.gov; Herbert A Thompson - thompoly@hotmail.com; Robert F Massung* - rfm2@cdc.gov

* Corresponding author

Published: 18 October 2007

BMC Microbiology 2007, 7:9| doi:|0.1|86/|47|-2/80-7-9|
Received: 23 March 2007

Accepted: 18 October 2007

This article is available from: http://www.biomedcentral.com/|47|-2/80/7/9|

(c) 2007 Denison et al; licensee BioMed Central Ltd.

This is an Open Access article distributed under the terms of the Creative Commons Attribution License (http://creativecommons.org/licenses/by/2.0), which permits unrestricted use, distribution, and reproduction in any medium, provided the original work is properly cited.

\begin{abstract}
Background: Coxiella burnetii contains the ISI/II transposase which is present 20 times in the Nine Mile phase I (9Mi/l) genome. A single PCR primer that binds to each IS element, and primers specific to a region $\sim 500$-bp upstream of each of the $20 \mathrm{ISIIII}$ elements were designed. The amplified products were characterized and used to develop a repetitive element PCR genotyping method.
\end{abstract}

Results: Isolates Nine Mile phase II, Nine Mile RSA 5I4, Nine Mile Baca, Scottish, Ohio, Australian QD, Henzerling phase I, Henzerling phase II, M44, KAV, PAV, Q238, QI95 and WAV were tested by PCR and compared to $9 \mathrm{Mi} / \mathrm{l}$. Sequencing was used to determine the exact differences in isolates which lacked specific IS elements or produced PCR products of differing size. From this data, an algorithm was created utilizing four primer pairs that allows for differentiation of unknown isolates into five genomic groups. Additional isolates (Priscilla Q 177, Idaho Q, Qiyi, Poker Cat, Q229 and Q172) and nine veterinary samples were characterized using the algorithm which resulted in their placement into three distinct genomic groups.

Conclusion: Through this study significant differences, including missing elements and sequence alterations within and near IS element coding regions, were found between the isolates tested. Further, a method for differentiation of $C$. burnetii isolates into one of five genomic groups was created. This algorithm may ultimately help to determine the relatedness between known and unknown isolates of $C$. burnetii.

\section{Background}

Coxiella burnetii is a gram-negative, obligate, intracellular bacterium that causes $\mathrm{Q}$ fever in humans. Infection occurs through inhalation of contaminated aerosols and fewer then ten organisms is sufficient to seed an infection [1]. Q fever can manifest as an acute infection characterized by a flu-like febrile illness, headache, and atypical pneumonia and the recommended treatment is doxycycline. A chronic infection is typified by endocarditis and/or hepatitis with poor prognosis [2].

Initial studies examining restriction fragment length polymorphisms (RFLP) in chromosomal and plasmid DNA from seven C. burnetii isolates revealed genetic heteroge- 
neity among the isolates [3]. In subsequent research, RFLP patterns analyzed via sodium dodecyl sulfate-polyacrylamide [4] or pulsed field gel electrophoresis [5] allowed for the creation of six distinct genomic groups (I through VI). Isolates from acute disease patients could be placed in genomic groups I, II and III, while groups IV and V contained isolates from chronic disease patients. Furthermore, isolates placed in genomic groups I, II and III were shown to contain the plasmid QpH1, group IV isolates to contain the plasmid QpRS, and group V isolates are plasmidless [5], though QpRS-homologous sequences are located in chromosomal genome sequences of the group $\mathrm{V}$ isolates $[6,7]$. The group VI isolates were initially reported to contain the plasmid QpDG, though newer experiments suggest these may actually possess QpH1, and QpDG containing isolates were shown to be avirulent in guinea pigs [8,9]. Additional studies have characterized a variety of isolates using RFLP/pulsed field gel electrophoresis with differing restriction enzymes [10], multispacer sequence typing [11], multiple loci variable number of tandem repeats analysis $[12,13]$, and microarrays [14] which have discriminated isolates into as many as 36 distinct genotypes.

With the completion of the genome sequence of Nine Mile Phase I (9Mi/I), 20 unique copies of the IS1111 transposase were identified [15]. Previous work describing the insertion sequence noted that the coding region of the transposase is bounded by two sets of terminal inverted repeats at each end, which were previously referred to as the inner and outer inverted repeats [16]. The inner inverted repeats are thought to represent the termini of the IS element. It is predicted that IS1111 forms a circular intermediate, whereby the inner inverted repeats are brought closer together to form a strong promoter which increases transposase expression. The outer inverted repeats are believed to be chromosomal sequences which form a stem-loop structure that acts as a recognition site for insertion of the IS element but are not part of the IS element itself. This chromosomal target sequence is present more than 50 times throughout the $9 \mathrm{Mi} / \mathrm{I}$ genome [17]. Sequence differences in the various copies of IS1111, most of which are present in the loop of the stem-loop recognition site, led to its further subdivision into IS1111A, IS1111B and IS1111C [16]. We sought to compare the locations of IS1111 in a variety of isolates of $C$. burnetii to that of the 20 copies present in $9 \mathrm{Mi} / \mathrm{I}$ using a PCR-based method. Because of the high copy number of the IS element in the C. burnetii genome and the unique regions adjacent to each element, this has proved to be a useful tool in differentiating isolates of $C$. burnetii into groups, much like the six genomic groups proposed previously $[4,5]$. We present an algorithm which distinguishes members from five of the six genomic groups using four primer pairs and a two-step PCR cycling program.

\section{Results}

PCR analysis included 14 isolates, 10 of which had previously been characterized as members of genetic groupings I through V [Nine Mile phase II (9Mi/II), Nine Mile RSA 514 (RSA 514), Ohio, Australian QD, Q195, Henzerling RSA 343 (Henz I), Henzerling, RSA 331 (Henz II), M44, KAV, PAV, Q238 and WAV], and 4 [Nine Mile Baca (9Mi/ Baca), Scottish, Q238 and WAV] which had not been genotyped [4]. Our repository lacks the group VI Dugway isolates, thus these were not included. The analysis was completed for each isolate for each of the 20 IS elements present in 9Mi/I using the consensus primer IS1111-1 paired with a unique primer upstream of each of the IS elements. The presence or absence of certain IS elements was generally consistent among members of a particular genogroup, but allowed their differentiation from members of other genogroups. The results from each of these 14 isolates are provided (see Additional file 1) along with the element's orientation and nucleotide position in $9 \mathrm{Mi}$ / I [GenBank: $\underline{\text { AE016828] }}$, and GenBank accession numbers for the sequences determined in this study.

\section{Genomic group I isolates}

9Mi/II, RSA 514, Ohio, and Australian QD appeared to have all 20 IS elements and their products were indistinguishable from each other and $9 \mathrm{Mi} / \mathrm{I}$ by this method. The isolates $9 \mathrm{Mi} /$ Baca and Scottish, which have not been genotyped previously, also had all 20 IS elements and were placed within this group.

\section{Genomic group II isolates}

PCR products amplified using DNA from isolates Henz I, Henz II, and M44 were identical in size to $9 \mathrm{Mi} / \mathrm{I}$ for 15 of the 20 IS elements $(2,3,5,6,7,8,9,11,12,13,15,16$, 17,18 , and 19). IS elements 14 and 20 were confirmed absent in these isolates by sequencing, though our M44 isolate also possessed a subpopulation which contained IS 14 (Fig 1). In regards to IS 14, the deletion totaled 1,374 bp, which corresponds exactly to the predicted length of the IS element [17]. Also within this region, Henz I and Henz II, but not M44, contain a point mutation. The IS 20 deletion in these three isolates totaled 2,246 bp.

Amplicons to IS elements 1 and 4 were present but of larger size than $9 \mathrm{Mi} / \mathrm{I}$. In all three group II isolates, indels consisted of small deletions and slightly larger insertions. The IS 10 region, however, was quite unique in that PCR products using primer pairs IS 10 and IS1111-1 produced a product of incorrect size. Upon amplification with primer pairs $1269 \mathrm{R}$ and $1273 \mathrm{R}$, which correspond to genes flanking the IS 10 element in $9 \mathrm{Mi} / \mathrm{I}$, products were obtained. Sequence data indicated that the site where the 


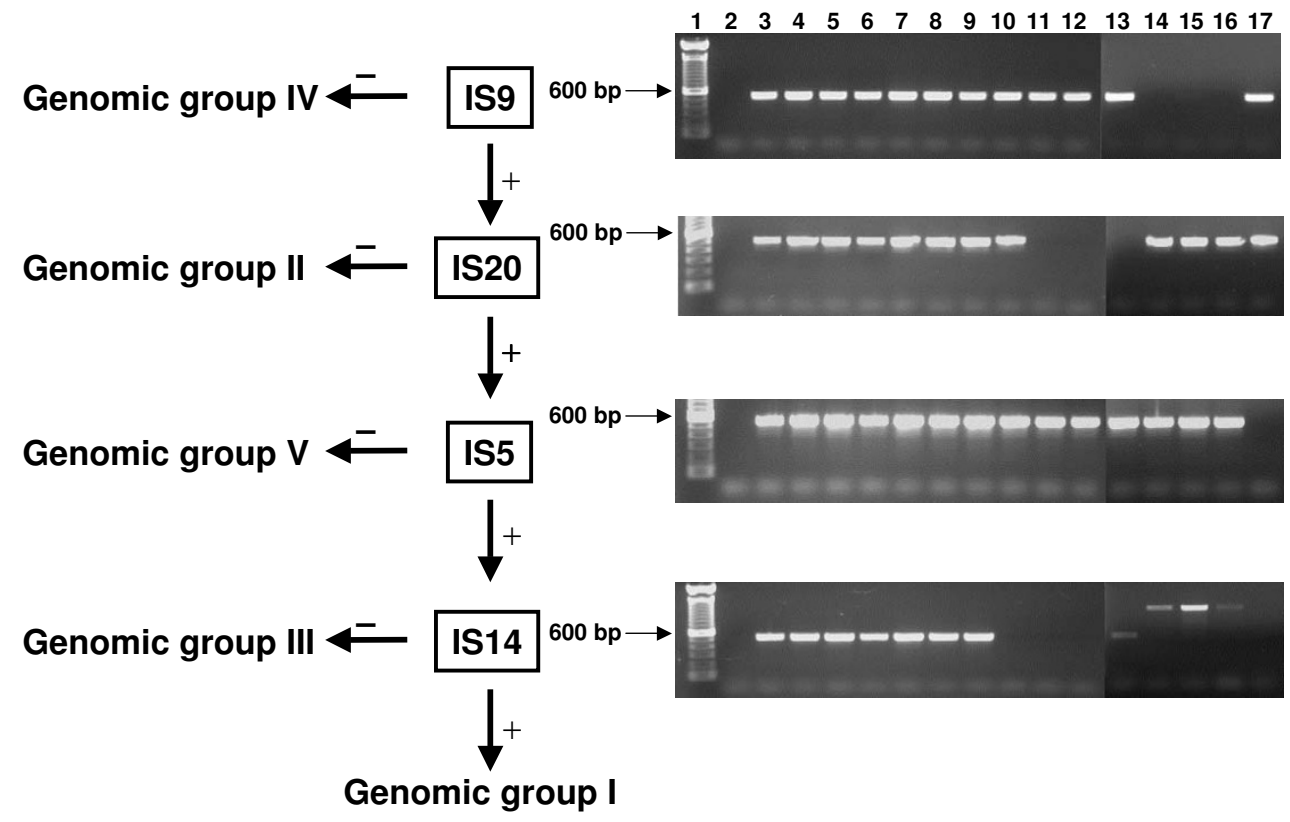

Figure I

Proposed algorithm flowchart and agarose gel photographs of corresponding PCR products. To discriminate isolates of $C$. burnetii using the algorithm shown, PCR is performed using the primer pairs depicted in boxes and negative PCR reactions allow for the discrimination of isolates into the genomic groups shown. Agarose gels of PCR products using primer pairs (from top to bottom) IS 9 and ISI III-I, IS 20 and ISIIIII-I, IS 5 and ISIIIII-I, and IS I4 and ISI III-I. Lanes I, I00 bp ladder; 2, negative control; 3, 9Mi/l; 4, 9Mi/II; 5, RSA 5I4; 6, 9Mi/Baca; 7, Scottish; 8, Ohio; 9, Australian QD; I0, QI95; II, Henz I; I2, Henz II; I3, M44; I4, KAV Q I54; I5, PAV Q I73; 16, Q238; I7, WAV.

IS 10 primer anneals is missing due to a deletion of 1,650 bp in these isolates, but that the IS element is indeed present.

\section{Genomic group III isolate}

A single isolate from genomic group III, Q195, was available in our repositories for analysis. There were no noticeable differences in amplicon sizes between 9Mi/I and Q195 for 15 of the 20 IS elements $(2,3,5,6,7,8,9,10$, $12,13,15,16,17,18$, and 19). The only IS element which Q195 lacked was IS 14. Sequencing of this region identified a genomic deletion of 1,374 bp.

Amplicons of larger size were produced to IS elements 1, 4 and 20. Upon sequencing of these PCR products, each was missing 3 nucleotides and an identical sequence of 26 nucleotides was inserted in its place in all cases. The PCR product for IS 11 was smaller for Q195 in comparison to $9 \mathrm{Mi} / \mathrm{I}$ and was found to be lacking $8 \mathrm{bp}$.

\section{Genomic group IV isolates}

Two isolates from chronic Q fever patients exhibiting endocarditis, KAV and PAV, have previously been placed into group IV [4]. Q238 is believed to be an isolate derived from the same patient as PAV, though it has not been gen- otyped previously. These three isolates were examined by PCR using the primer pairs to each of the 20 IS elements described above. No amplicon size differences were seen for 10 of the IS elements $(2,5,6,8,11,12,13,17,19$ and 20) when compared to $9 \mathrm{Mi} / \mathrm{I}$.

Analysis of IS 7 and IS 9 in the KAV, PAV and Q238 isolates showed that both of these IS elements were missing. Sequencing revealed the absence of 1,458 bp and 1,341 bp, respectively, compared to the IS 7 and IS 9 elements in the 9Mi/I genome. Similarly, IS elements 14, 15, and 16 were missing in each of the genomic group IV isolates because of identical deletions of 1,374 bp.

No products of the correct size were obtained from isolates KAV, PAV and Q238 with primers for IS 10. A long PCR product obtained using primers 1269R and 1273R was sequenced and an IS element was found in this region, although the annealing site for the IS 10 primer was eliminated. The sequence of DNA to which the IS element is connected corresponds to $9 \mathrm{Mi} / \mathrm{I}$ nucleotide positions 1222860-1222964. The sequence is then interrupted by 133 bp of sequence that does not match sequence in $9 \mathrm{Mi} / \mathrm{I}$, but does match sequence found in the Dugway 7E9-12 strain [GenBank: AAQI00000000]. 
Products of IS 18-specific PCR in each of the three genomic group IV isolates appeared to be slightly larger than that of $9 \mathrm{Mi} / \mathrm{I}$. Upon sequencing of the KAV product, however, this appeared to be a consequence of primer mismatch to another IS element in the genome. The sequence data obtained [GenBank: DQ882594] corresponded to an IS element and a novel sequence not present in the $9 \mathrm{Mi} / \mathrm{I}$ genome. However, this sequence was found in the Dugway 7E9-12 strain. Using long PCR to flanking genes and DNA from the three isolates, no products were obtained, though PCR of each of the flanking genes (data not shown) indicated they are present somewhere in the genome, suggesting that in these isolates, this region of the genome has undergone rearrangement compared to $9 \mathrm{Mi} / \mathrm{I}$.

PCR to IS elements 1, 3, and 4 produced products from $\mathrm{KAV}, \mathrm{PAV}$ and Q238 differing in size relative to $9 \mathrm{Mi} / \mathrm{I}$. These variations were due to numerous indels ranging from $1 \mathrm{bp}$ to $32 \mathrm{bp}$. The indels in both IS 1 and IS 3 resulted in frame shifts in the predicted IS1111 encoded protein.

\section{Genomic group $\boldsymbol{V}$ isolate}

WAV had not been characterized previously but was hypothesized to be a member of genomic group $\mathrm{V}$, as it could be distinguished from each of the other isolates at a variety of IS elements. It was indistinguishable from $9 \mathrm{Mi} /$ I for 12 of the 20 IS elements $(2,3,6,8,9,10,11,12,13$, 16,17 , and 18). Six nucleotides were missing in WAV in the products amplified from IS elements 1, 4, and 20 when compared to $9 \mathrm{Mi} / \mathrm{I}$ and an identical 21-bp sequence was present in its place in each case.

No amplicon was generated using primers to IS elements $5,7,14,15$ and 19. Sequencing confirmed the absence of IS 5, 14 and 15 due to deletions of $1,374 \mathrm{bp}$, and of IS 7 due to a 1,458 bp deletion. Lastly, PCR to IS 19 using DNA from WAV did not result in an amplicon. Long PCR using primers $1960 \mathrm{R}$ and $1693 \mathrm{R}$ resulted in a product larger than that from $9 \mathrm{Mi} / \mathrm{I}$ (data not shown). Sequencing elucidated that a rearrangement had occurred in this area of the WAV genome, but was not pursued further as it was evident that an IS element was no longer present in this portion of the WAV genome.

\section{Creation and testing of an algorithm to differentiate $\mathrm{C}$. burnetii strains}

Using the information gained above, an algorithm was derived which discriminated each of the five genomic groups of isolates tested (Fig. 1). Because only group IV heart valve isolates (KAV, PAV and Q238) lacked products to IS 9, Henz I, Henz II, and M44 (group II) lacked IS 20, and WAV (group V) lacked IS 5, PCR to these isolates could be used to discriminate these groups. Isolates posi- tive to each of these IS elements could then be further tested using PCR to IS 14, which separates Q195 (group III) isolates from group I isolates. PCR to IS 14 also serves to confirm that group II, IV and V isolates, which also lack this IS element, are grouped appropriately.

Further, each of the primer pairs used in the algorithm was tested using a two-step PCR program, and all products were successfully amplified (Fig. 1). The algorithm and the two-step PCR was then tested with additional isolates of $C$. burnetii and veterinary samples previously shown to be PCR positive for the agent. This included two isolates previously characterized, Priscilla Q177 (group IV) and Q229 (group V), four isolates of unknown genomic group (Idaho Q, Qiyi, Poker Cat and Q172), and nine veterinary samples from a single outbreak in 2005 on a goat ranch in Colorado. As expected, Priscilla Q177 and Q229 could be differentiated appropriately due to the lack of PCR products when using primers to IS 9 and IS 5, respectively. Q172 could also be placed into group V based on its lack of product to IS 5. PCR to IS 14 was also negative in these isolates, confirming that Priscilla Q177, Q229, and Q172 lacked this IS element as predicted. The remaining isolates (Idaho Q, Qiyi and Poker Cat) produced PCR products with all primer pairs and therefore were identical to $9 \mathrm{Mi}$ / I in our assay and placed within group I (data not shown). Six of the veterinary samples lacked PCR products to IS 9 and IS 14, placing them in genomic group IV. Three of the veterinary samples, one from placenta and two from feces, did not give PCR products using the above conditions. Since these three samples were positive for C. burnetii when tested with a real time-PCR assay that amplifies the IS1111 element, it was suspected that an inhibitor was present. By using a master mix that has demonstrated increased sensitivity in our hands (PuReTaq Ready-To-Go PCR Beads, GE Healthcare, Fairfield, CT) in lieu of Qiagen Taq Master Mix, PCR products were successfully obtained such that these samples were also shown to be members of genomic group IV.

\section{Discussion}

In this study, PCR amplification and DNA sequencing of the IS1111 transposase elements in the genome of C. burnetii and their surrounding sequence has been used to extensively characterize these elements in multiple isolates, and as target regions for inter- and intra-genomic analysis. In some isolates, IS element PCR resulted in products either larger or smaller than expected based on the $9 \mathrm{Mi} / \mathrm{I}$ genome and these were generally due to small indels in the isolates tested. Numerous IS elements present in the $9 \mathrm{Mi} / \mathrm{I}$ genome were absent in the isolates evaluated, mostly due to large deletions. Of the 25 deletions which could be completely characterized by sequencing, 16 were 1,374 in length. Larger deletions were detected at IS $20(2,246 \mathrm{bp})$ in the group II isolates 
and at IS 7 ( $1,458 \mathrm{bp})$ in the group IV and V isolates, while a smaller deletion (1,341 bp) was detected at IS 9 in the group IV isolates. The 1,374-bp length corresponds exactly with the predicted length of the IS element [17], demonstrating that the IS element has likely failed to integrate into these sites thus far. The presence of larger deletions may indicate that the IS element inserted into certain sites and subsequently excised, taking additional genomic sequence with it or causing genomic rearrangements when doing so. Numerous nucleotide polymorphisms were also seen in the stem-loop portion of the IS insertion target sites (data not shown) which may render the sites unrecognizable by the transposase, or alternatively, the IS element simply may not have inserted into these IS recognition sites. The sequence data demonstrate that, where polymorphisms were noted, the stem of the stem-loop structure is most times preserved while changes are evident in the loop region. An understanding of target site recognition and insertion mechanisms will help to determine the importance of the nucleotides within the loop region. If it is found that the overall structure but not specific nucleotide sequence is key to recognition, the division of IS1111 into IS1111A, IS1111B and IS1111C, as previously suggested based on sequence variation, may be unnecessary [16].

Analysis of the M44 isolate was complicated by evidence of a mixed population when analyzing IS 14 . This result could indicate that the isolate is truly a mixed population, which is quite possible since it was never cloned. Previous work from our laboratory supports this hypothesis, as prior analysis of this isolate by an indirect immunofluorescence assay demonstrated epitopes reactive with both phase I- and phase II-specific antibodies [18]. However, this result could also occur if IS1111 is actively transposing in the genome, in which case circular intermediates should be detectable. PCR to determine if circular forms of IS1111 were present in 9Mi/I did not result in a product, though the linear form of IS1111 appeared to be expressed as judged by reverse transcriptase-PCR analysis (data not shown). It may be that expression of this linear form leads to an actively transposing IS element at low levels that we could not detect by PCR. The IS element appears rather stable, however, in that all 9Mi/I derived isolates have IS elements identically placed with no copies having been eliminated, though additional copies could be present and would not be detected by the methods used in this study. Certainly further research into the mechanism and frequency of IS1111 transposition is warranted.

The inter-genomic comparisons based on our IS element analysis showed very good correlation with RFLP analysis that was previously used to divide C. burnetii isolates into six distinct genomic groups [4]. Therefore, this analysis was used to develop a PCR-based genotyping assay which identifies an isolate as within one of the genomic groups, I through $\mathrm{V}$. The assay was effective in discriminating genomic groupings in veterinary samples as well as purified isolates of $C$. burnetii. While most veterinary samples could be differentiated using the conditions initially set forth, an inhibitor was found to block amplification in three of the veterinary samples, as PCR products to all IS elements, even those conserved in all isolates tested, were not produced. Inhibition was easily circumvented by using a different master mix. It should be noted that inhibition occurred with both of the fecal samples that were tested. DNA from fecal samples is typically more difficult to amplify due to the presence of inhibitors and other factors, and if DNA from feces will be used for genotyping, extractions should be performed using protocols designed especially for feces [19]. Therefore, it is recommended that a control utilizing primers to a conserved IS element or a housekeeping gene also be included in the run to check for inhibition of the reaction. The DNA from any group I isolate should be used as a positive size control in applications of the algorithm, as group I isolates produce a product with all four algorithm primer pairs. Further, negative PCR results to any of the IS elements in the algorithm should also be verified by sequencing the region to ensure that mutations or deletions in primer binding regions are not at fault and that the IS element is truly lacking from this region. Sequencing of PCR products elucidated single nucleotide polymorphisms in IS element regions (data not shown) which could distinguish KAV from PAV, though no differences were observed between Henz I and Henz II or PAV and Q238. Sequencing was also effective at determining which IS elements were being amplified, and in some cases, sequences not found in the $9 \mathrm{Mi} / \mathrm{I}$ genome were identified next to IS elements. It is interesting to note that in the group IV isolates, IS 10 and IS 18 primers annealed to sequences present in the Dugway 7E9-12 genome but not in Nine Mile. The origin of the Dugway strain and how the group IV isolates came to share genomic sequence with this strain, evolutionarily speaking, is intriguing. While other genomic changes undetectable by this method are surely present in these isolates, which would allow for further discrimination of isolates into a larger number of genotypes, the ability to quickly identify members of genomic groups I through $\mathrm{V}$ is useful. Perhaps a combination of rapid PCR-based methods such as the algorithm presented here, and VNTR (variable number tandem repeats) analysis $[12,13]$ or MLS (multispacer sequence) typing [11] for potentially higher resolution, will prove optimal.

It is interesting to note that the Qiyi isolate, typed as genomic group I in this study, was reportedly isolated from the bone marrow of a patient with chronic Q fever [20]. This represents a relatively novel finding, and sup- 
ports a previous report that identified an isolate characterized as within genomic group I from a chronically infected human in France [10]. In contrast, most isolates from chronically infected humans have been classified into genomic groups IV and V. Clearly, further examination of the genomic composition and evolutionary relatedness of isolates from both acute and chronic human infections is warranted.

\section{Conclusion}

This study has begun to shed new light on the variations of IS elements within the genome of multiple isolates and the mechanism of IS element insertion in the C. burnetii genome. It also presents an algorithm which may provide a useful tool for grouping unknown isolates. Further, with the additional use of sequencing in these regions, a multitude of information can be learned about new isolates which may be used to determine their relatedness to known isolates of C. burnetii.

\section{Methods}

\section{Bacterial isolates and veterinary samples}

The isolates studied, their source, and passage history are shown in Table 1 . Isolates 9Mi/I, 9Mi/II, RSA 514, 9Mi/ Baca, Scottish, Ohio, Australian QD, Q195, Henz I, Henz
II, M44, KAV Q154, PAV Q173, Q238 and WAV, which represent members of genomic groups I through $\mathrm{V}$, were initially used to create the algorithm presented below. Isolates Priscilla Q177, Idaho Q, Qiyi, Poker Cat, Q229 and Q172 were employed to test the algorithm, as were nine veterinary samples from a single outbreak at a goat ranch in Colorado in 2005. Of the nine samples, five were taken from placental tissue, two from feces, and two from milk.

\section{DNA isolation, PCR and sequencing analysis}

DNA was extracted from purified bacteria or crude yolk sac preparations using the QIAamp DNA Mini Kit (Qiagen, Valencia, CA). DNA was extracted from veterinary samples using the QIAamp DNA Blood Kit (Qiagen), following the manufacturers protocol for extraction from tissue samples. A PCR primer (IS1111-1) was designed to anneal to a sequence conserved in all IS elements within the $9 \mathrm{Mi} / \mathrm{I}$ genome, and was paired with each of 20 primers selected to bind to unique sequences approximately 500 bp upstream from each of the 20 IS elements. The sequence of each primer used in this study is listed in Table 2, along with the predicted amplicon size. For initial screening as well as algorithm testing, PCR reactions were performed in a $25 \mu$ l volume using the Qiagen Taq PCR Master Mix kit, $0.2 \mu \mathrm{M}$ of each primer, and $0.5 \mu \mathrm{l}$ of tem-

Table I: Original source and passage history of $C$. burnetii isolates used in this study.

\begin{tabular}{|c|c|c|c|c|c|}
\hline \multicolumn{2}{|c|}{ Genomic group } & \multirow[b]{2}{*}{ Plasmid type } & \multirow[b]{2}{*}{ Isolate (Phase) ${ }^{c}$} & \multirow[b]{2}{*}{ Original source } & \multirow[b]{2}{*}{ Passage history ${ }^{d}$} \\
\hline RFLPa & $|\mathrm{S} / / /| \mathrm{PCR}^{b}$ & & & & \\
\hline I & 1 & QpHI & $9 \mathrm{Mi} / \mathrm{l} / \mathrm{C7}(\mathrm{I})$ & Montana, tick, 1935 & 307GP/ITC/IEP \\
\hline I & 1 & QpHI & $9 \mathrm{Mi} / \mathrm{II} / \mathrm{C} 4$ (II) & Montana, tick, 1935 & 304GP/90EP/ITC/4EP \\
\hline I & I & $\mathrm{QpHI}$ & 9Mi/RSA 514 (II) & Montana, tick, 1935 & 307GP/ITC/IEP/343 days GP/5EP \\
\hline I & I & QpHI & Ohio (I) & Ohio, cow's milk, 1956 & $5 \mathrm{EP} / 2 \mathrm{GP} / 2 \mathrm{EP}$ \\
\hline I & I & QpHI & Australian QD (II) & Australia, human blood, $\sim 1939$ & I77EP \\
\hline NDe & i & $\mathrm{QpHI}$ & Scottish (ND) & Unknown & Unknown \\
\hline ND & 1 & QpHI & Idaho Q (ND) & Unknown & Unknown \\
\hline ND & I & ND & 9Mi/Baca (II) & Montana, tick, 1935 & 307GP/ITC/IEP/409I days TC \\
\hline II & II & QpHI & Henzerling RSA 343 (I) & Italy, human blood, 1945 & 6GP/25EP/IGP/4EP \\
\hline II & ॥ & $\mathrm{QpHI}$ & Henzerling RSA 33I (II) & Italy, human blood, 1945 & 6GP/25EP/IGP/36EP \\
\hline II & II & $\mathrm{QpHI}$ & M44 (II) & Greece, human blood, $\sim 1945$ & IGP/86EP/20GP/50MP/5EP \\
\hline III & III & QpHI & Q195 (ND) & Idaho, goat & Unknown \\
\hline IV & IV & QpRS & KAV QI54 (I) & Oregon, human heart valve, 1976 & IGP/3EP \\
\hline IV & IV & QpRS & PAV QI73 (I) & California, human heart valve, 1979 & $2 \mathrm{EP}$ \\
\hline IV & IV & QpRS & Priscilla QI77 (I) & Montana, goat cotyledon, 1980 & Unknown \\
\hline ND & IV & QpRS & Q238 (ND) & Human heart valve, 1979 & 3EP \\
\hline V & $\vee$ & Plasmidless & Q229 (I) & Human heart valve, 1982 & Unknown \\
\hline ND & $\mathrm{V}$ & ND & WAV (ND) & Human heart valve & Unknown \\
\hline ND & $\vee$ & ND & Q 172 (ND) & Human placenta & $3 M P / 2 E P$ \\
\hline ND & I & ND & Qiyi (ND) & China, human bone marrow, 1962 & Unknown \\
\hline ND & 1 & ND & Poker Cat (ND) & Nova Scotia, 1987 & Unknown \\
\hline
\end{tabular}

${ }^{a}$ As determined in previous studies [4, 5].

${ }^{b}$ As determined in this study.

c Phase of isolate as determined by complement block titration, M.G. Peacock, Rocky Mountain Laboratories, Hamilton, Montana [4].

${ }^{d}$ Numbers indicate passage in: GP, guinea pig; TC, tissue culture; EP, embryonated eggs; MP, mice

e Not determined 
Table 2: Primers, their target, sequence, primer pairings and predicted amplicon size of primer pair.

\begin{tabular}{|c|c|c|c|}
\hline Target & Primer name & Sequence (5' to $\left.3^{\prime}\right)$ & Paired primer set and predicted amplicon size \\
\hline Conserved for each IS element & $|S| I|I-|$ & ACTGCGTTGGGATACCCATC & $\mathrm{NA}^{a}$ \\
\hline ISI & ISI & TAATGGGCGACCAAGTCGA & ISIIIII-I; $50 \mid$ bp \\
\hline IS2 & IS2 & GGCTGAATGAATGCCTTCCA & ISIIII-I; $50 \mid$ bp \\
\hline IS3 & IS3 & AAAAAGACCCTTCAGGTAATGGAA & ISIIII-I; 477 bp \\
\hline IS4 & IS4 & TTCCGCCATGACCAACTTC & ISIIII-I; 519 bp \\
\hline \multirow[t]{2}{*}{ IS5 } & IS5 & GTCGGTCAACGTCGTCACAT & ISIIII-I; 515 bp \\
\hline & $556 \mathrm{~F}$ & AACCGTGGCTGAGCGATC & IS5; NA \\
\hline IS6 & IS6 & CCATTTGCAATAGCCGTGAA & ISIIII-I; 477 bp \\
\hline \multirow[t]{2}{*}{ IS7 } & IS7 & GCGGTTTTGGTACAATACTGTCATT & ISIIII-I; $57 \mid$ bp \\
\hline & $1091 \mathrm{~F}$ & TGCAGGACATATTGTTGATGTCAC & IS7; NA \\
\hline IS8 & IS8 & GGCAAGGATGACGAATGGAT & ISIIII-I; 47I bp \\
\hline \multirow[t]{2}{*}{ IS9 } & IS9 & GCCTCAGCCGATTTCGAG & ISIIII-I; 492 bp \\
\hline & $1219 \mathrm{~F}$ & CCCGAAGGTATCCATAACTCTGG & IS9; NA \\
\hline \multirow[t]{3}{*}{ ISIO } & ISIO & GTGCGGCATTCGTGGTAGA & ISIIII-I; 48I bp \\
\hline & I269R & GATTTCCATCGACGTCCGC & I273R; NA \\
\hline & I273R & TTCAGTTTATAGCGGCAGGACC & ISIIII-I; NA \\
\hline ISII & $|S| I$ & AAAGCCGTTCCCTACCGTG & ISIIII-I; 48I bp \\
\hline$|S| 2$ & ISI2 & ACGTAGTTTGGAAAGGATACCCG & ISIIII-I; 482 bp \\
\hline ISI3 & ISI3 & GATGAGCGTCCACCACTGAA & ISIIII-I; 537 bp \\
\hline \multirow[t]{2}{*}{ ISI4 } & ISI4 & TGCTACCAACAGACTTACGGCA & ISIIII-I; 48I bp \\
\hline & I70IR & TAAACACGAGGCGCTAGGTCA & ISI4; NA \\
\hline \multirow[t]{2}{*}{ ISI5 } & ISI5 & AACGCCCATGTGAGAAACATC & ISIIII-I; 53I bp \\
\hline & $1718 \mathrm{~F}$ & ACGGGTGTTCAAGCAGCC & ISI5; NA \\
\hline \multirow[t]{2}{*}{ ISI6 } & ISI6 & ATGACTTCTGATAGGGACTGTGCTC & ISIIIII-I; 5II bp \\
\hline & $1761 \mathrm{~F}$ & GCCGTTGATCTTGCCGATAA & ISI6; NA \\
\hline ISI7 & ISI7 & CGAATCGCATTGGCACAGT & ISIIII-I; 477 bp \\
\hline ISI8 & ISI8 & CGTTTGCTGCTATACGACATGAA & ISIIII-I; 532 bp \\
\hline \multirow[t]{4}{*}{ ISI9 } & ISI9 & GTCGCCCAGGAGTGTTTCTG & ISIIII-I; 53I bp \\
\hline & I960R & ACAAAGACGACTCAGTTCGGC & 1963R; NA \\
\hline & I963R & CGATGTCTGCCTTTAGGAGGTC & I960R; NA \\
\hline & 1963R-seq & GTAGAAAGAGTCTCTCTTG & NA \\
\hline \multirow[t]{3}{*}{ IS20 } & IS20 & ACGTCAATTACATCGAGCATTCA & ISIIIII-I; 470 bp \\
\hline & $1987 \mathrm{~F}$ & AGGGCCATCTTGACCTGGA & I993F; NA \\
\hline & $1993 \mathrm{~F}$ & CGCACTGAGCGCATTGAC & I987F; NA \\
\hline
\end{tabular}

${ }^{a}$ Not applicable

plate DNA. For initial screenings, PCR cycling conditions consisted of an initial denaturation at $95^{\circ} \mathrm{C}$ for $3 \mathrm{~min}, 35$ cycles each consisting of $30 \mathrm{~s}$ at temperatures of $95^{\circ} \mathrm{C}$, $55^{\circ} \mathrm{C}$, and $72^{\circ} \mathrm{C}$, and a final extension for $7 \min$ at $72^{\circ} \mathrm{C}$. Reactions testing the algorithm (IS elements 5, 9, 14 and 20) utilized $2 \mu \mathrm{l}$ of template DNA and were subjected to cycling conditions of an initial denaturation at $95^{\circ} \mathrm{C}$ for 3 min, 40 cycles each consisting of $30 \mathrm{~s}$ at temperatures of $95^{\circ} \mathrm{C}$ and $68^{\circ} \mathrm{C}$, and a final extension for $7 \mathrm{~min}$ at $72^{\circ} \mathrm{C}$. PCR products were visualized using ethidium bromidestained $1.2 \%$ agarose gels.

For isolates which lacked specific IS elements, the upstream primer was paired with additional PCR primers (see Table 2) to adjacent downstream genes based on the 9Mi/I genome sequence [GenBank: AE016828]. In some instances, new upstream primers were also needed to determine the extent of the deleted DNA (IS10, IS18 and IS19), sometimes resulting in large PCR amplicons. When
PCR amplicons were predicted to be larger than $1.5 \mathrm{~kb}$, long PCR was performed. These reactions were carried out in $25 \mu \mathrm{l}$ volumes using Extensor High-Fidelity PCR Master Mix (ABgene Inc. USA, Rochester, NY), $0.2 \mu \mathrm{M}$ of each primer, and $0.5 \mu \mathrm{l}$ of template DNA. PCR cycling conditions consisted of an initial denaturation at $94^{\circ} \mathrm{C}$ for 2 min, 10 cycles each consisting of $94^{\circ} \mathrm{C}$ for $10 \mathrm{sec}, 55^{\circ} \mathrm{C}$ for $30 \mathrm{sec}$, and $68^{\circ} \mathrm{C}$ for $8 \mathrm{~min}$, followed by 20 cycles each consisting of $94^{\circ} \mathrm{C}$ for $10 \mathrm{sec}, 55^{\circ} \mathrm{C}$ for $30 \mathrm{sec}$, and $68^{\circ} \mathrm{C}$ for $8 \mathrm{~min}+10 \mathrm{sec} /$ cycle, with a final extension for $7 \mathrm{~min}$ at $68^{\circ} \mathrm{C}$. Products were visualized using ethidium bromidestained $0.8 \%$ agarose gels.

In instances where amplicons were of a different size than those present in $9 \mathrm{Mi} / \mathrm{I}$ or where products were obtained using primers to genes adjacent to a missing IS element, DNA sequencing was performed. PCR products obtained above were processed through a DNA Clean \& Concentrator-5 column (Zymo Research Corp., Orange, CA) and 
eluted in $16 \mu$ l of water. Sequencing reactions were performed with $1 \mu \mathrm{l}$ of purified PCR product, one of the primers used to generate that PCR product, and the BigDye Terminator v3.1 Cycle Sequencing kit (Applied Biosystems, Foster City, CA). Unincorporated dye terminators were removed with a DyeEx 2.0 Spin Kit (Qiagen), the eluate was dried in a vacuum centrifuge, and the pellet suspended in $10 \mathrm{ul}$ of Hi-Di formamide (Applied Biosystems). Sequencing reactions were analyzed in an ABI Prism 3100 Genetic Analyzer, and the Genetics Computer Group Wisconsin Package Version 10.3 (Accelrys Inc., San Diego, CA) was used for subsequent analysis of DNA sequences.

\section{Authors' contributions}

AMD carried out all experimental elements of the study and drafted the manuscript. HAT and RFM conceived the study, participated in its design and helped to draft the manuscript. All authors have read and approved the final manuscript.

\section{Additional material}

\section{Additional file 1}

Presence (+) or absence (-) of IS elements in various isolates of C. burnetii; Table includes a description of each of the 20 IS elements and notes whether the element was present or absent in each of the isolates tested, as well as Genbank accession numbers for deletions and mutations discovered during this study.

Click here for file

[http://www.biomedcentral.com/content/supplementary/14712180-7-91-S1.xls]

\section{Acknowledgements}

We thank Jonathan Arzt (Veterinary Diagnostic Laboratory, Colorado State University) for providing the veterinary samples used in this study and Rachael Priestley for extracting DNA from the veterinary samples.

This research was supported in part by the appointment of A.M.D. to the ASM Post-Doctoral ResearchFellowship Program in Infectious Diseases and Public Health Microbiologysupported by the Centers for Disease Control and Prevention and administered by the American Society for Microbiology, as well as the appointment of A.M.D. to the Research Participation Program at the Centers for Disease Control and Prevention, Coordinating Center for Infectious Diseases, Division of Viral and Rickettsial Diseases, administered by the Oak Ridge Institute for Science and Education through an interagency agreement between the U.S. Department of Energy and CDC.

The findings and conclusions in this report are those of the authors and do not necessarily represent the views of the CDC or the Department of Health and Human Services.

\section{References}

I. Benenson AS, Tigertt WD: Studies on $\mathbf{Q}$ fever in man. Trans Assoc Am Physicians 1956, 69:98-104.
2. Raoult D, Marrie T, Mege J: Natural history and pathophysiology of $\mathbf{Q}$ fever. Lancet Infect Dis 2005, 5:219-226.

3. Vodkin MH, Williams JC, Stephenson EH: Genetic heterogeneity among isolates of Coxiella burnetii. J Gen Microbiol 1986, 132:455-463.

4. Hendrix LR, Samuel JE, Mallavia LP: Differentiation of Coxiella burnetii isolates by analysis of restriction-endonucleasedigested DNA separated by SDS-PAGE. J Gen Microbiol I99I, 137:269-276.

5. Heinzen R, Stiegler GL, Whiting LL, Schmitt SA, Mallavia LP, Frazier ME: Use of pulsed field gel electrophoresis to differentiate Coxiella burnetii strains. Ann N Y Acad Sci 1990, 590:504-5I3.

6. Willems H, Ritter M, Jager C, Thiele D: Plasmid-homologous sequences in the chromosome of plasmidless Coxiella burnetii Scurry Q2 17. J Bacteriol 1997, 179:3293-3297.

7. Savinelli EA, Mallavia LP: Comparison of Coxiella burnetii plasmids to homologous chromosomal sequences present in a plasmidless endocarditis-causing isolate. Ann N Y Acad Sci 1990, 590:523-533.

8. Stoenner HG, Lackman DB: The biologic properties of Coxiella burnetii isolated from rodents collected in Utah. Am J Hyg 1960, 71:45-51.

9. Jager $\mathrm{C}$, Lautenschlager S, Willems H, Baljer G: Coxiella burnetii plasmid types QpDG and QpHI are closely related and likely identical. Vet Microbiol 2002, 89:161-166.

10. Jager C, Willems H, Thiele D, Baljer G: Molecular characterization of Coxiella burnetii isolates. Epidemiol Infect 1998, 120:157-164.

II. Glazunova O, Roux V, Freylikman O, Sekeyova Z, Fournous G, Tyczka J, Tokarevich N, Kovacava E, Marrie TJ, Raoult D: Coxiella burnetii genotyping. Emerg Infect Dis 2005, I I:I2 I I-12 I I.

12. Arricau-Bouvery N, Hauck Y, Bejaoui A, Frangoulidis D, Bodier CC, Souriau A, Meyer H, Neubauer H, Rodolakis A, Vergnaud G: Molecular characterization of Coxiella burnetii isolates by infrequent restriction site-PCR and MLVA typing. BMC Microbiol 2006, 6:38.

13. Svraka S, Toman R, Skultety L, Slaba K, Homan WL: Establishment of a genotyping scheme for Coxiella burnetii. FEMS Microbiol Lett 2006, 254:268-274.

14. Beare PA, Samuel JE, Howe D, Virtaneva K, Porcella SF, Heinzen RA: Genetic diversity of the $Q$ fever agent, Coxiella burnetii, assessed by microarray-based whole-genome comparisons. J Bacteriol 2006, 188:2309-2324.

15. Seshadri R, Paulsen IT, Eisen JA, Read TD, Nelson KE, Nelson WC, Ward NL, Tettelin H, Davidsen TM, Beanan MJ, Deboy RT, Daugherty SC, Brinkac LM, Madupu R, Dodson RJ, Khouri HM, Lee KH, Carty HA, Scanlan D, Heinzen RA, Thompson HA, Samuel JE, Fraser CM, Heidelberg JF: Complete genome sequence of the Q-fever pathogen Coxiella burnetii. Proc Natl Acad Sci USA 2003, 100:5455-5460.

16. Hoover TA, Vodkin MH, Williams JC: A Coxiella burnetii repeated DNA element resembling a bacterial insertion sequence. J Bacteriol 1992, 174:5540-5548.

17. Partridge SR, Hall RM: The ISI I I I family members IS432 I and IS5075 have subterminal inverted repeats and target the terminal inverted repeats of Tn2I family transposons. J Bacteriol 2003, 185:637I-6384.

18. Denison AM, Massung RF, Thompson HA: Analysis of the O-antigen biosynthesis regions of phase II Isolates of Coxiella burnetii. FEMS Microbiol Lett 2007, 267: 102-107.

19. Zhang BW, Li M, Ma LC, Wei FW: A widely applicable protocol for DNA isolation from fecal samples. Biochem Genet 2006, 44:494-503.

20. Ning Z, Yu SR, Quan YG, Xue Z: Molecular characterization of cloned variants of Coxiella burnetii isolated in China. Acta Virol 1992, 36:173-183. 